


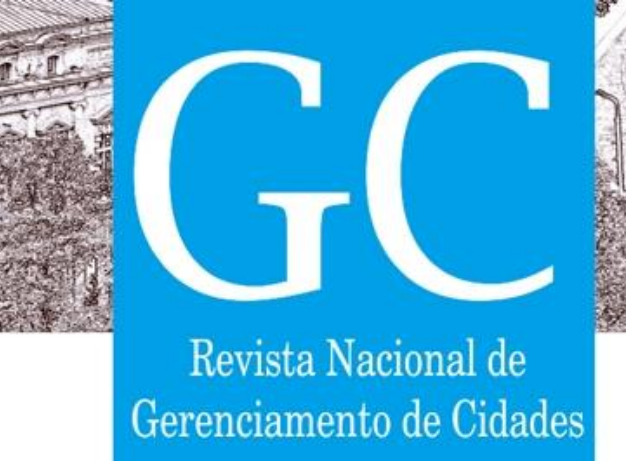

cenários foram definidos: Cenário 1 - Urbanização Desordenada Hipotética; Cenário 2 Barragem; Cenário 3 - Parques Fluviais; Cenário 4 - Barragem + Parques Fluviais. O índice REFLU foi, então, aplicado.

O Índice REFLU é uma ferramenta que permite avaliar a qualidade de um rio urbano pela obtenção de uma medida quantitativa (Veról et al., 2019). O resultado do índice para diferentes cenários pode ser facilmente comparado, analisando a eficácia das medidas de requalificação fluvial propostas. Ele é calculado a partir da soma de diversos efeitos relacionados à qualidade fluvial. O resultado obtido para o REFLU deve estar compreendido entre 0 e 1 , sendo que quanto mais próximo de 1 , melhor o resultado.

Originalmente, o REFLU era composto por quatro subíndices, como apresentado em Veról et al. (2019), mas ao longo do desenvolvimento dessa pesquisa, a sua estrutura foi revisada, com o intuito de facilitar a sua aplicação e torná-lo mais conciso. Nesta nova concepção, o REFLU é composto por três subíndices, a saber: Estado Geral da Bacia (EGB), composto por três indicadores, Permeabilidade (P), Saneamento Adequado (SA) e Margens Ocupadas pela Urbanização (MOUrb); Conectividades (C), composto por três indicadores, Conectividade Transversal (CT), Conectividade Longitudinal (CL) e Conectividade Vertical (CV) e Redução do Risco Hidráulico (RRH) que é ele próprio, também, o indicador.

A Figura 2 ilustra esta nova estrutura, definida para o REFLU, e a Equação 1 apresenta sua formulação final, adotada neste trabalho. 


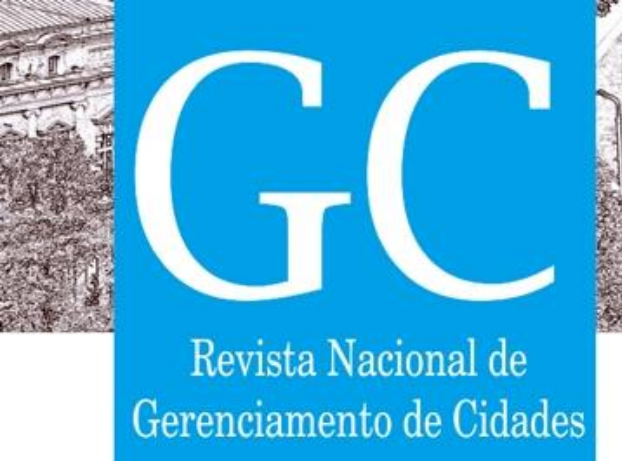

Figura 1: Nova estrutura definida para o REFLU

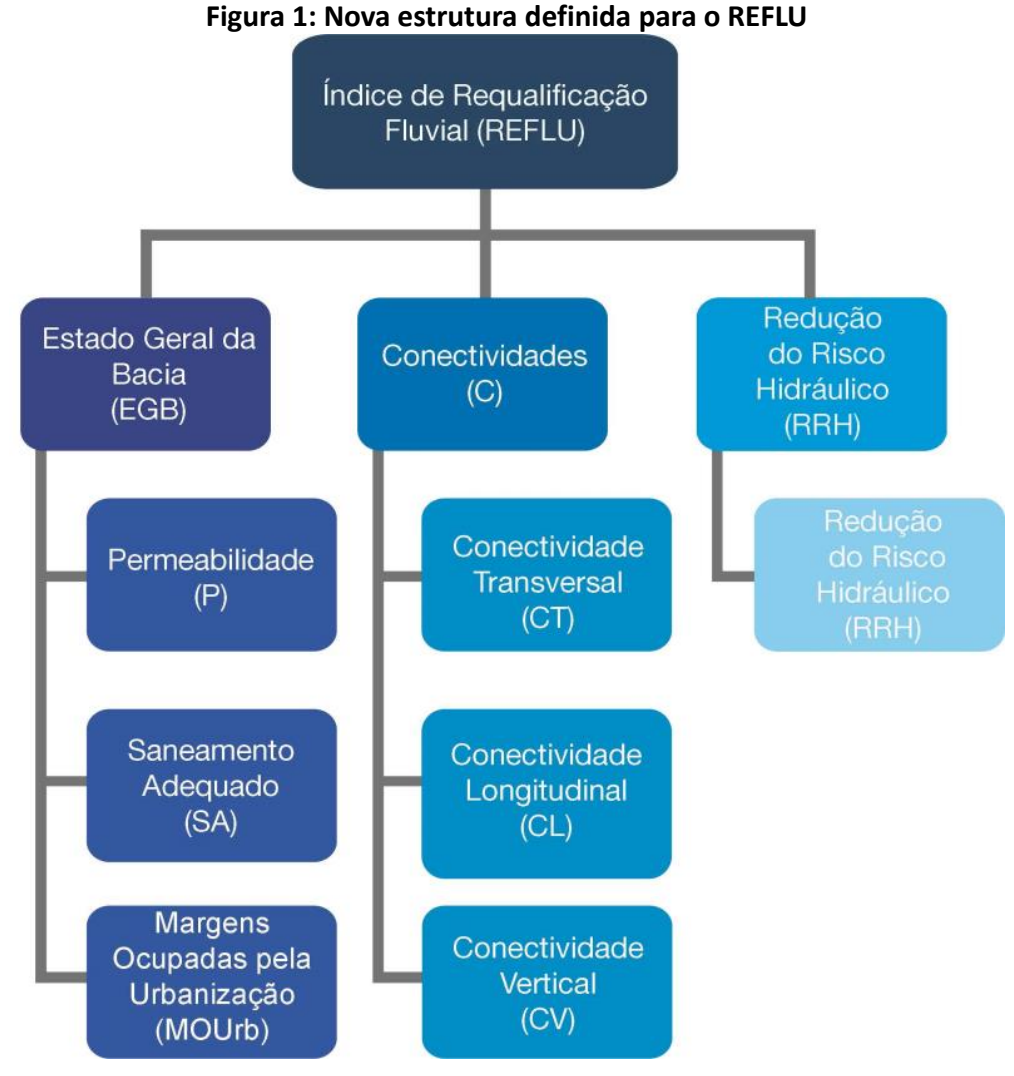

$R E F L U=\left(E G B \cdot p^{E G B}\right)+\left(C \cdot p^{c}\right)++\left(R R H \cdot p^{R R H}\right)$

(Equação 1)

onde:

$R E F L U$ : Índice de Requalificação Fluvial;

$E G B$ : Subíndice "Estado Geral da Bacia", relativo aos impactos da ocupação da bacia hidrográfica relacionados ao uso do solo e a gestão de recursos hídricos. Indicadores: "Permeabilidade", "Saneamento Adequado" e Margens Ocupadas pela Urbanização;

$C$ : Subíndice "Conectividades", relativo ao nível de conectividade do curso d'água principal. Indicadores: "Conectividade Transversal", "Conectividade Longitudinal" e "Conectividade Vertical";

RRH: Subíndice "Redução do Risco Hidráulico", relativo aos alagamentos nas áreas urbanizadas. Relaciona-se o volume de alagamento no pico da cheia ao volume total precipitado.

$p^{E G B}, p^{C}, p^{R R H}$ :Pesos associado aos subíndices, atribuídos em função de sua importância relativa. O somatório dos pesos " $p i$ " deve resultar 1. 


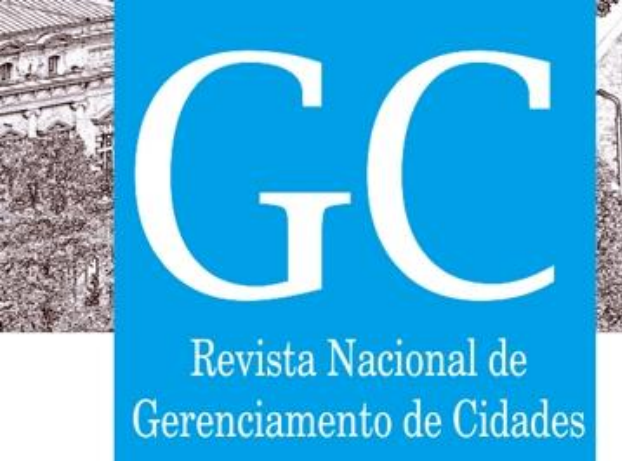

A ferramenta básica de simulação hidrodinâmica adotada para mapeamento de alagamentos em áreas urbanas (um dos dados de entrada do REFLU) foi o MODCEL (Miguez et al., 2017), concebido a partir do conceito de células de escoamento e já utilizado em diversos outros estudos (Miguez et al, 2019; Oliveira et al, 2019; Rezende et al, 2020), inclusive na Baixada Fluminense (Oliveira et al, 2017; Jacob et al, 2019) onde se situa o estudo de caso. No MODCEL, as células atuam como compartimentos homogêneos, integrando o espaço da bacia e permitindo definir redes de escoamento complexas, bidimensionais. Nesse contexto, combinase, verticalmente, uma rede de escoamentos superficiais com uma rede subterrânea de escoamentos, relativa às galerias de drenagem, numa evolução para um esquema que pode ser considerado, topologicamente, como pseudo tri-dimensional.

\section{ESTUDO DE CASO}

Para estudo de caso foi escolhida a bacia dos Rios Pilar-Calombé, no município de Duque de Caxias, Região Metropolitana do Rio de Janeiro. A bacia dos Rios Pilar-Calombé foi objeto de estudo no recém-publicado Plano Estratégico de Desenvolvimento Urbano e Integrado da Região Metropolitana do Rio de Janeiro (Quanta-Lerner, 2017), elaborado entre 2015 e 2018 e que teve como pontos chave a implantação de infraestrutura e aprimoramento da gestão do saneamento ambiental, por meio de infraestrutura de manejo de águas pluviais, coleta e tratamento de esgoto sanitário e destinação adequada dos resíduos sólidos, assim como a garantia do abastecimento regular de água em toda a Região Metropolitana do Rio de Janeiro. Além disso, também visa garantir a gestão das áreas protegidas e a conservação da biodiversidade por meio de Planos de Manejo e gestão das Unidades de Conservação que se encontram próximas ao Arco Metropolitano. O uso e ocupação do solo de todas as áreas no entorno do Arco deve ser disciplinado, e os vazios urbanos devem ser tratados como zonas de amortecimento.

O Arco Metropolitano (Figura 2) articula quatro importantes rodovias federais sem passar pelo núcleo urbano, fazendo a ligação entre dois polos econômicos: o polo petroquímico do COMPERJ e o Porto de Itaguaí. Ao longo do Arco, podem ser observadas diferentes unidades de paisagem (Tângari et al., 2012). Há uma forte presença de indústrias de grande porte, o que atrai empreendimentos residenciais para a região. Também são identificados vazios urbanos, causados pela demarcação de Áreas de Proteção Ambiental. A maior parte do território urbano metropolitano urbanizado está ao sul do Arco. Como impactos negativos da construção do Arco, pode ser destacado que a rodovia funciona como um obstáculo, aumentando a segregação em um tecido urbano que já é muito fragmentado. O estabelecimento de novas moradias no entorno da rodovia muitas vezes acontece de forma irregular e desordenada. Nesse cenário, os espaços livres devem ser aproveitados para projetos de revitalização urbana, assim como para o manejo de águas pluviais urbanas. 


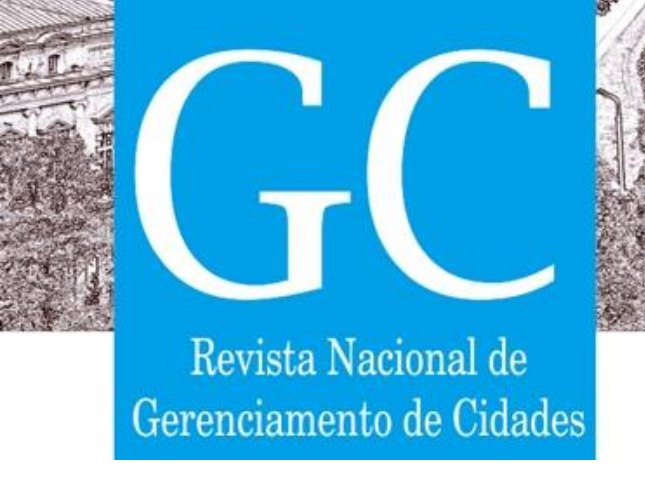

ISSN 2318-8472

v. 08, n. 59,2020

Figura 4: Mapa de Inundação - Situação Atual, Bacia dos Rios Pilar-Calombé

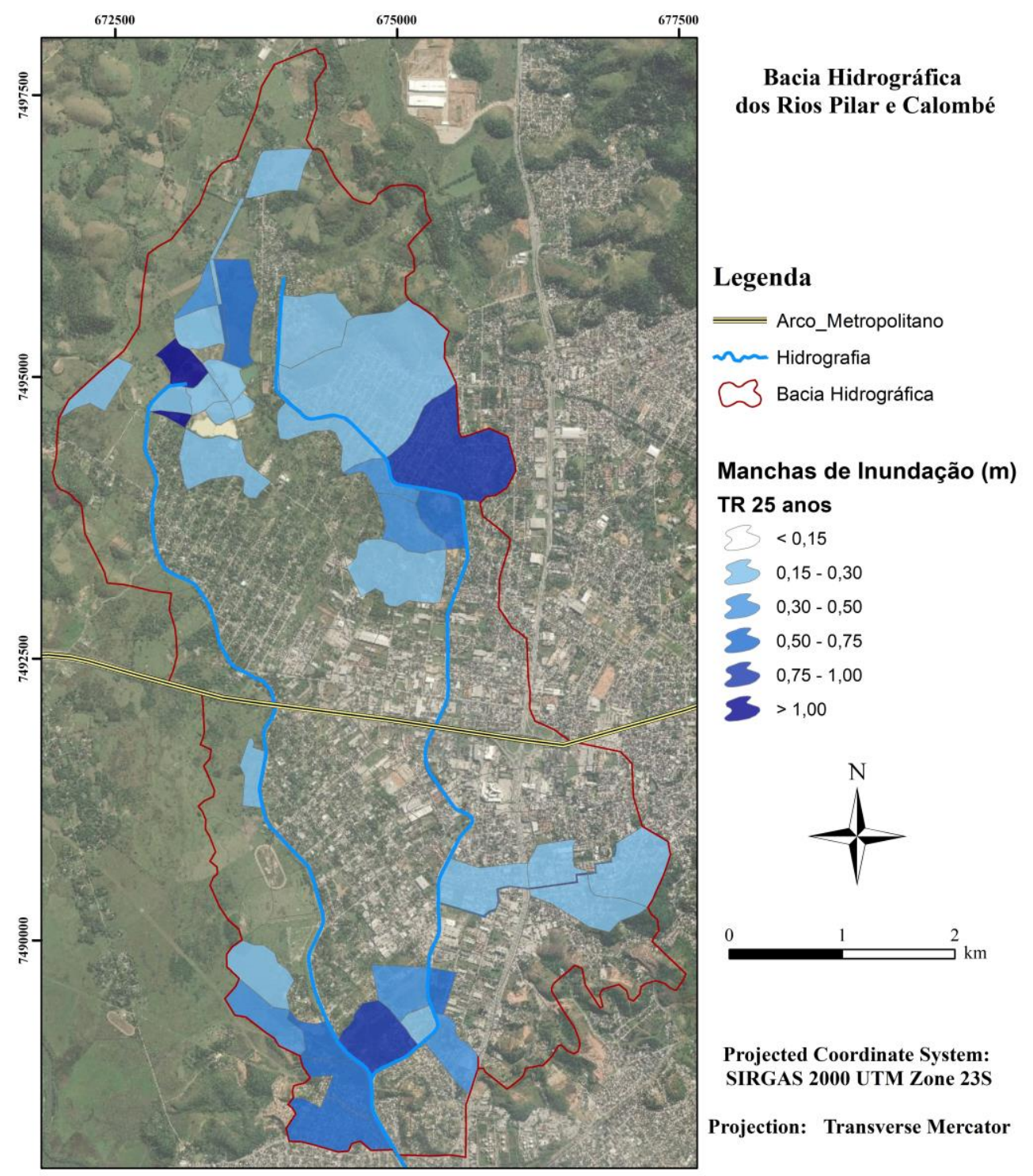

\subsection{Cenário 1 - Urbanização Desordenada Hipotética}

Para o Cenário 1, foi simulada uma ocupação desordenada hipotética, que poderia vir a acontecer devido à presença do Arco Metropolitano. Foi visto que a ocupação ao sul do Arco é mais densa, então é considerado, aqui, que esse padrão de ocupação vai se espalhar também para a área ao norte do Arco (Figura 5a). 


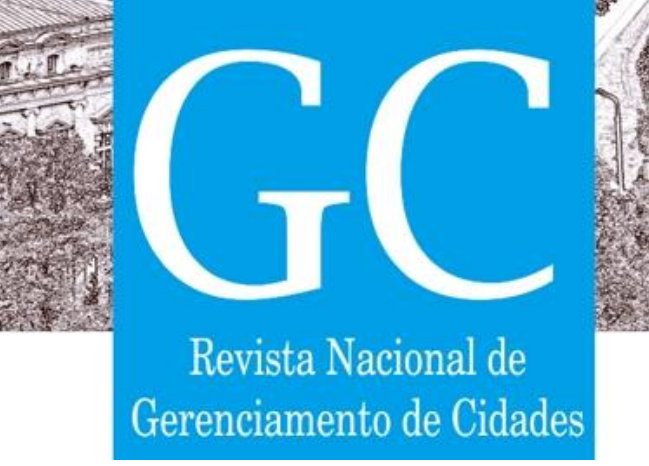

\subsection{Barragem}

O segundo cenário proposto envolve a criação de uma barragem posicionada junto ao Arco Metropolitano do Rio de Janeiro, criando uma zona de alagamento controlado ao norte, ou seja, à montante dos Rios Pilar-Calombé, conforme apresenta a Figura 5b. Pretende-se que essa área seja ocupada por sítios de produção agropecuária, como está indicado no Plano Metropolitano (Quanta-Lerner, 2017) e por casas adaptadas para os eventos de cheias.

Barragens são interrupções transversais ao sentido dos rios, que criam uma zona de inundação controlada à montante e reduzem o volume de água à jusante, reduzindo o risco hidráulico. Como aspectos negativos, elas prejudicam a transferência de matéria (água, sedimentos e matéria orgânica) e existe um risco de rompimento associado.

\subsection{Parques Fluviais}

O terceiro cenário propõe a implantação de parques fluviais inundáveis. Eles teriam 15 metros de largura e ocupariam ambas as margens dos rios, nos trechos demarcados na Figura $5 \mathrm{c}$. Esses espaços permitem o estabelecimento da calha secundária do rio, alocando o volume de cheias e impedindo que elas causem danos à cidade. Os parques fluviais protegem a zona ribeirinha de ocupações irregulares, restauram as várzeas, recompõem a vegetação, protegem as margens contra erosão e reduzem os picos de cheias. Diferente do cenário de barragem, que propõe uma medida tradicional para controle de inundações, esse traz uma solução resiliente que melhora a resposta urbana aos eventos de pluviosidades intensas, além de respeitar a dinâmica do rio e buscar recuperar o estado natural da bacia.

Esse cenário foi elaborado com base no trabalho de (Rezende, 2010), que propôs uma série de medidas para o controle de cheias na bacia, incluindo a criação de parques fluviais, e as avaliou através da ferramenta MODCEL.

\subsection{Barragem + Parques Fluviais}

Foi também proposto um cenário que combinasse as duas propostas anteriores. Nele, a barragem e os parques fluviais acontecem simultaneamente, atuando em trechos diferentes da bacia hidrográfica (a barragem mais a montante e os parques a jusante), conforme ilustra a Figura $5 d$. 


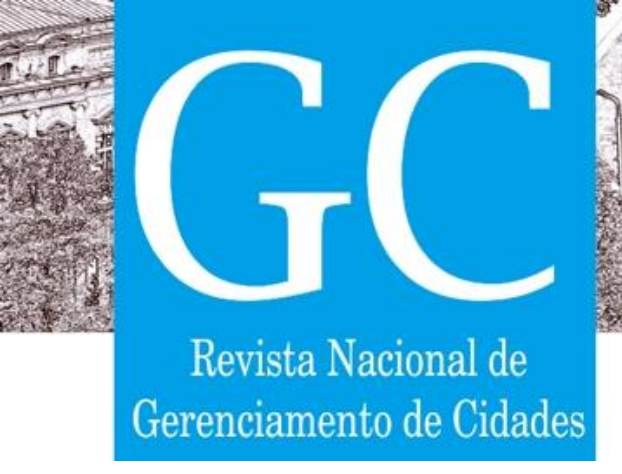

Figura 6: Gráficos com os resultados do REFLU por subíndice para todos os cenários simulados.
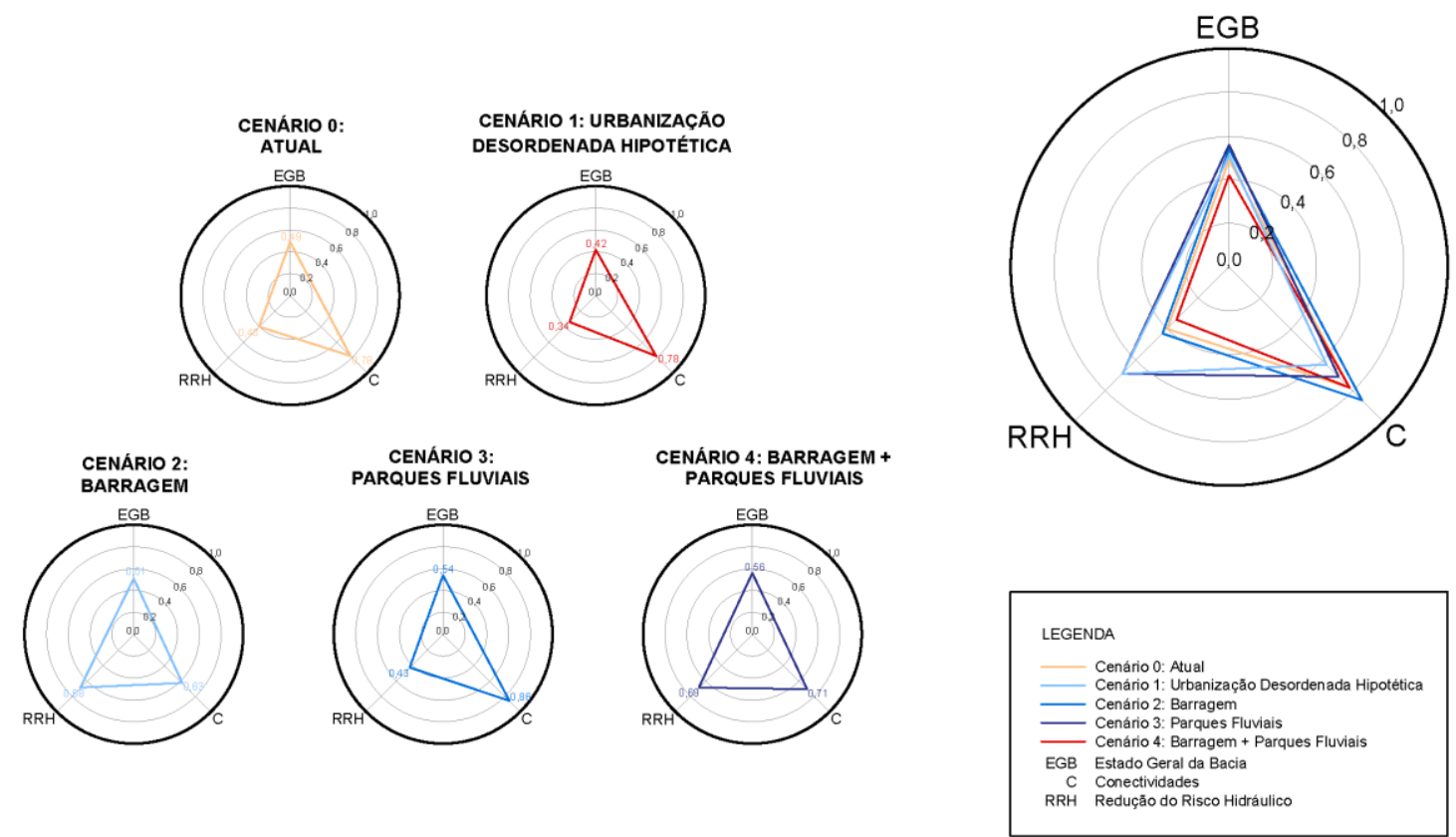

Figura 7: Gráficos com os resultados do índice REFLU.

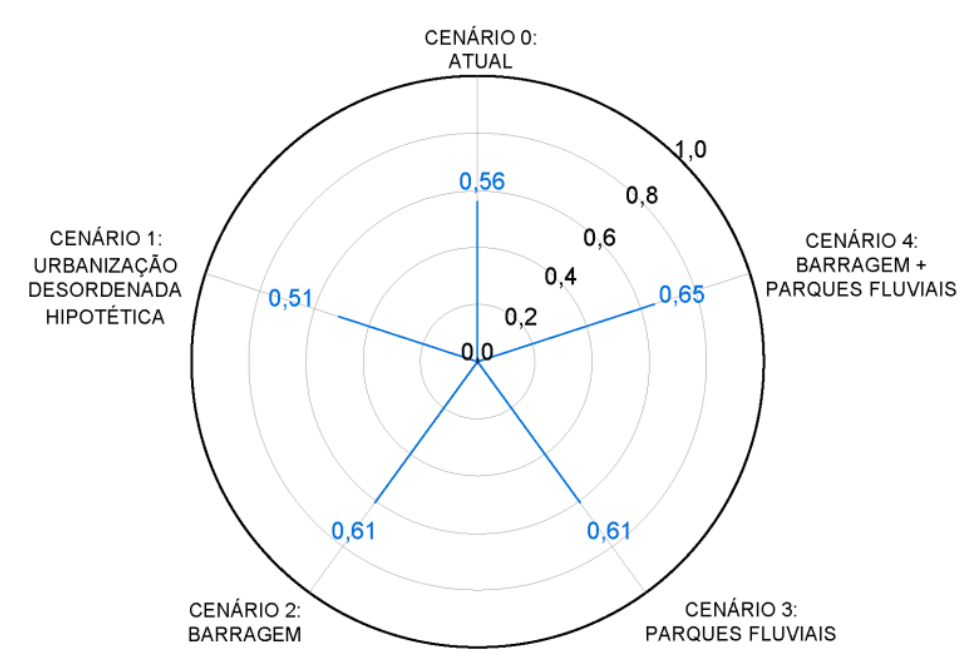

\section{CONCLUSÃO}




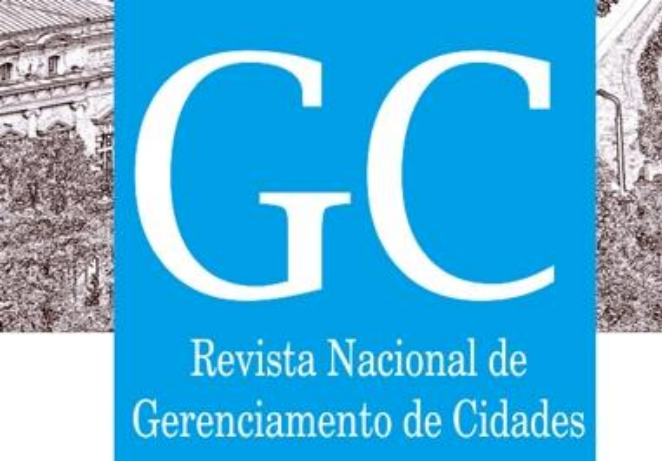

O êxito em tornar sustentável o crescimento das cidades está intimamente relacionado a um planejamento urbano consistente, que seja capaz de compatibilizar as demandas urbanas com aquelas do sistema natural. O presente estudo ratificou a importância desta relação a partir da comparação de diferentes cenários de desenvolvimento urbano na bacia dos Rios PilarCalombé, Região Metropolitana do Rio de Janeiro, no que concerne às relações entre água e cidade.

Para este estudo, foram concebidos cenários futuros, aplicados a bacia. Os cenários abordados consideraram uma urbanização sem controle; a introdução de uma barragem para controle de cheias; a introdução de parques fluviais ao longo dos rios, com viés sustentável, reservando espaço para o amortecimento de cheias; e a combinação destes em um quarto cenário.

Por meio de modelagem matemática e da aplicação do Índice de Requalificação Fluvial foram avaliados os impactos de cada cenário proposto e comparados à Situação Atual. Assim, conforme as expectativas, a Situação Atual refletiu o comportamento atual da bacia, enquanto os demais indicaram melhoria, com destaque para o Cenário que combina a solução de parques fluviais com a introdução de uma barragem de controle de cheias, como previsto no PEDUI/RMRJ. Foi também proposto um cenário de urbanização desordenada, que apresentou uma inundação de forma generalizada, enquanto os demais cenários apresentaram uma inundação mais controlada.

O REFLU, aplicado à bacia dos Rios Pilar-Calombé, foi capaz de comparar o desempenho de cada projeto de forma abrangente, considerando o controle de inundação, a melhoria ambiental e o estado geral da bacia, traduzindo em resultados de simples interpretação os ganhos e perdas para cada cenário. Em relação ao controle de inundações e ganhos urbanos, a barragem foi muito efetiva para o controle de inundações, mas afetou a conectividade, com perda de valor ambiental. Os parques fluviais, por sua vez, apesar de articular os aspectos ambientais com a urbanização, têm uma limitação de volume para controlar os alagamentos. Outra questão relevante foi que o estado geral da bacia variou pouco, apesar dos esforços, mostrando que a questão do saneamento básico, com ênfase para o esgotamento sanitário, que não foi abordado nesse trabalho, é uma questão crucial na discussão da cidade. Ressalta-se que os melhores resultados, em termos médios, foram os que combinaram as medidas mais tradicionais com soluções de infraestrutura verde.

Pode-se dizer ao final deste estudo, que a requalificação fluvial pode ser uma importante ferramenta para o controle de enchentes, enquanto resgatando qualidade para o sistema fluvial e gerando oportunidades de revitalização urbana. Essa alternativa deve ser conjugada com técnicas compensatórias de drenagem urbana para suporte na recuperação de características do ciclo hidrológico natural. O resultado final desta composição de projeto vem minimizar riscos e aumentar a resiliência da cidade. 


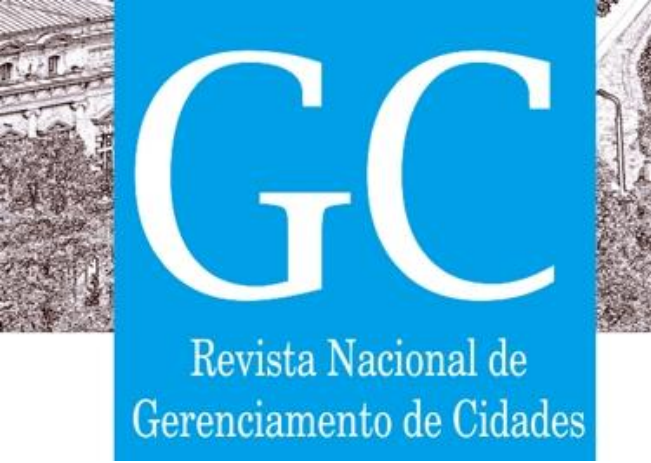

MIGUEZ, Marcelo Gomes et al. Urban flood simulation using MODCEL-an alternative quasi-2D conceptual model. Water, v. 9(6), p.445, 21 jun. 2017. https://doi.org/10.3390/w9060445

MIGUEZ, Marcelo Gomes et al. A framework to support the urbanization process on lowland coastal areas: Exploring the case of Vargem Grande-Rio de Janeiro, Brazil. Journal of Cleaner Production, v. 231, p. 1281-1293, 2019.

MORANDI, Bertrand et al. How is success or failure in river restoration projects evaluated? Feedback from French restoration projects. J. Environ. Manage, v. 137, p. 178-188, 1 mai. 2014. https://doi.org/10.1016/j.jenvman.2014.02.010

OLIVEIRA, Antonio Krishnamurti Beleño et al. O papel da concepção de espaços livres no planejamento da paisagem, com foco na drenagem urbana e controle de inundações: um estudo de caso para a Cidade dos Meninos em Duque de Caxias/RJ. In: XXII Simpósio Brasileiro de Recursos Hídricos, Florianópolis. Anais do XXII Simpósio Brasileiro de Recursos Hídricos, 2017.

OLIVEIRA, Antonio Krishnamurti Beleño. An alternative flood model calibration strategy for urban watersheds: the case study of Riohacha, Colombia. Water Science and Technology, n. 79(11), p. 2095-2105, 2019.

PAN, Baozhu et al. A review of ecological restoration techniques in fluvial rivers. International Journal of Sediment Research, v. 31, p. 110-119, jun. 2016. https://doi.org/10.1016/j.ijsrc.2016.03.001

QUANTA-LERNER, Consórcio. Plano Estratégico de Desenvolvimento Urbano Integrado da Região Metropolitana do Rio de Janeiro (PDUI/RMRJ). Rio de Janeiro: Câmara Metropolitana de Integração Governamental (CMIG), 2017.

PRIOR, Jonathan. Urban river design and aesthetics: a river restoration case study from the UK. J. Urban Des, v. 21, p. 512-529, 31 mai. 2016. https://doi.org/10.1080/13574809.2016.1187557

REZENDE, Osvaldo Moura. Avaliação de Medidas de Controle de Inundações em um Plano de Manejo Sustentável de Águas Pluviais Aplicado à Baixada Fluminense. Rio de Janeiro: COPPE-UFRJ, 2010.

REZENDE, Osvaldo Moura; MIGUEZ, Marcelo Gomes; VERÓL, Aline Pires. Manejo de Águas Urbanas e sua Relação com o Desenvolvimento Urbano em Bases Sustentáveis Integradas - Estudo de Caso dos Rios Pilar-Calombé, em Duque de Caxias. Rio de Janeiro, n.18, p. 149-163, 2013.

REZENDE, Osvaldo Moura et al. Mapping The Flood Risk to Socioeconomic Recovery Capacity through a Multicriteria Index. Journal of Cleaner Production, v. 225, n. 120251, 10 mai. 2020.

ROZOS, E.; MAKROPOULOS, C., MAKSIMOVIĆ, Č. Rethinking urban areas: An example of an integrated blue-green approach. Water Sci. Technol. Water Supply, v. 13(6), p. 1534-1542, 12 set. 2013. https://doi.org/10.2166/ws.2013.140

SAYERS, P. et al. Flood Risk Management: A Strategic Approach, Strategic Water Management, v. 203, 2013.

$\mathrm{SCHUCH}$, Gemma et al. Water in the city: Green open spaces, land use planning and flood management - An Australian case study. Land Use Policy, v. 63, p. 539-550, abr. 2017. https://doi.org/10.1016/j.landusepol.2017.01.042 


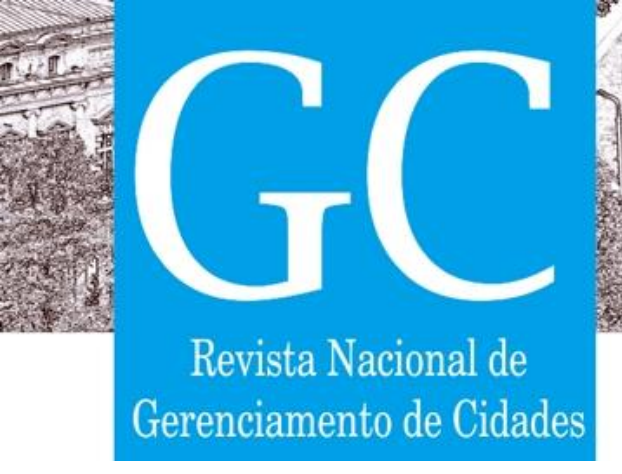

TÂNGARI, Vera Regina; REGO, Andrea Queiroz; MONTEZUMA, Rita de Cássia Martins. O Arco Metropolitano do Rio de Janeiro - integração e fragmentação da paisagem metropolitana e dos sistemas de espaços livres de edificação. PROARQ-FAU/UFRJ, Rio de Janeiro, 2012.

VERÓL, Aline Pires. Requalificação Fluvial Integrada ao Manejo de Águas Urbanas para Cidades Mais Resilientes. Rio de Jeaneiro: COPPE-UFRJ, 2013.

VERÓL, Aline Pires et al. The urban river restoration index (URRIX) - A supportive tool to assess fluvial environment improvement in urban flood control projects. J. Clean. Prod. v. 239, 1 dez. 2019. https://doi.org/https://doi.org/10.1016/j.jclepro.2019.118058 